\title{
Does sleep facilitate the consolidation of allocentric or egocentric representations of implicitly learned visual-motor sequence learning?
}

\author{
Jeremy Viczko, ${ }^{1,2}$ Valya Sergeeva, ${ }^{1,2}$ Laura B. Ray, ${ }^{1}$ Adrian M. Owen, ${ }^{1,2}$ \\ and Stuart M. Fogel ${ }^{1,2,3,4,5}$ \\ ${ }^{1}$ The Brain \& Mind Institute, Western University, London, Ontario N6A 5B7, Canada; ${ }^{2}$ Department of Psychology, Western University, \\ London, Ontario N6A 5C2, Canada; ${ }^{3}$ School of Psychology, University of Ottawa, Ottawa, Ontario K1N 6N5, Canada; ${ }^{4}$ The Royal's \\ Institute for Mental Health Research, Ottawa, Ontario K1Z 7K5, Canada; ${ }^{5}$ University of Ottawa Brain and Mind Research Institute, \\ Ottawa, Ontario K1H 8M5, Canada
}

\begin{abstract}
Sleep facilitates the consolidation (i.e., enhancement) of simple, explicit (i.e., conscious) motor sequence learning (MSL). MSL can be dissociated into egocentric (i.e., motor) or allocentric (i.e., spatial) frames of reference. The consolidation of the allocentric memory representation is sleep-dependent, whereas the egocentric consolidation process is independent of sleep or wake for explicit MSL. However, it remains unclear the extent to which sleep contributes to the consolidation of implicit (i.e., unconscious) MSL, nor is it known what aspects of the memory representation (egocentric, allocentric) are consolidated by sleep. Here, we investigated the extent to which sleep is involved in consolidating implicit MSL, specifically, whether the egocentric or the allocentric cognitive representations of a learned sequence are enhanced by sleep, and whether these changes support the development of explicit sequence knowledge across sleep but not wake. Our results indicate that egocentric and allocentric representations can be behaviorally dissociated for implicit MSL. Neither representation was preferentially enhanced across sleep nor were developments of explicit awareness observed. However, after a 1-wk interval performance enhancement was observed in the egocentric representation. Taken together, these results suggest that like explicit MSL, implicit MSL has dissociable allocentric and egocentric representations, but unlike explicit sequence learning, implicit egocentric and allocentric memory consolidation is independent of sleep, and the timecourse of consolidation differs significantly.
\end{abstract}

[Supplemental material is available for this article.]

One of the most important functions of sleep is to support learning and memory (for reviews, see Maquet 2001; Smith 2001; Doyon et al. 2009b; Fogel and Smith 2011; Rasch and Born 2013; Stickgold and Walker 2013; Stickgold 2013; Tononi and Cirelli 2014). A period of sleep, compared with wake, is known to enhance and transform labile memories into enduring long-term storage (Korman et al. 2003; Walker 2005; Sirota and Buzsáki 2007; Takehara-Nishiuchi and McNaughton 2008; Squire 2009; Giuditta 2014), enhance the performance of newly learned skills (Fischer et al. 2002; Peigneux et al. 2004; Bergmann et al. 2008; Doyon et al. 2009a; Albouy et al. 2013; Fogel et al. 2015), and can even promote conscious insight into otherwise unconscious knowledge (Wagner et al. 2004; Drosopoulos et al. 2005; Gómez et al. 2006; Yordanova et al. 2008; Payne et al. 2009). These enhancements in memory and skill performance after sleep are a phenomenon collectively referred to as "sleep-dependent memory consolidation." Sleep has been found to be important for motor memory consolidation, in particular, when learned explicitly (Fischer et al. 2002; Cohen et al. 2005; Morin et al. 2008; Albouy et al. 2013, 2015; Debas et al. 2014). Interestingly, sleep does not enhance all aspects of learning and memory equally. Rather, sleep preferentially supports the consolidation of dissociable memory representations (e.g., spatial aspects versus motor aspects) of proce-

\section{Corresponding author: sfogel@uottawa.ca}

Article is online at http://www.learnmem.org/cgi/doi/10.1101/lm.044719.116. dural motor skills (Cohen et al. 2005; Albouy et al. 2013, 2015). Importantly, it is not known whether this applies to motor skills that are learned without conscious knowledge. This may help to elucidate whether sleep is involved in the consolidation of implicit motor sequence learning (MSL), for which the precise role of sleep, if any, remains to be conclusively resolved.

MSL is multifaceted, comprising both allocentric (spatial) and egocentric (motor) frames of reference. Here we explore the possibility that sleep may only be involved in the consolidation of the allocentric representation for implicit MSL (Albouy et al. 2013; Cohen et al. 2005). Using neuroimaging Albouy et al. (2015) isolated the neural activation patterns specific to allocentric and egocentric performance for explicit MSL. They found that the spatial and motor representations rely on the hippocampo- and striatocortical networks, respectively. Importantly, sleep-dependent performance enhancement emerged for the hippocampal-dependent memory trace, whereas performance was only maintained for the striatal-dependent memory trace, regardless of whether sleep or wake took place during the retention interval. These results suggest that the hippocampus and the striatum support, respectively, the

2018 Viczko et al. This article is distributed exclusively by Cold Spring Harbor Laboratory Press for the first 12 months after the full-issue publication date (see http://learnmem.cshlp.org/site/misc/terms.xhtml). After 12 months, it is available under a Creative Commons License (Attribution-NonCommercial 4.0 International), as described at http://creativecommons.org/licenses/by-nc/ $4.0 \%$. 
allocentric and egocentric representation of an explicitly learned motor sequence during the learning process; with hippocampal activity related to sleep-dependent memory consolidation.

At present, there is little consensus about whether newly learned implicit motor sequences are enhanced over a period of sleep when compared with wake. Whether a similar dissociation (e.g., spatial versus motor) exists for implicitly learned sequences, is unclear, and could help to resolve existing controversies in the literature concerning whether sleep actively consolidates implicit motor sequence learning (Maquet et al. 2000; Peigneux et al. 2003; Cohen et al. 2005; Destrebecqz et al. 2005; Fischer et al. 2006; Urbain et al. 2013) or not (Keisler et al. 2007; Song et al. 2007; Nemeth et al. 2010, 2012; Hallgató et al. 2013; Pan and Rickard 2015). Some of the earlier studies using classic motor learning tasks, such as the serial reaction time task (SRTT) (Nissen and Bullemer 1987), have provided evidence for sleep-dependent memory consolidation of implicit motor learning, in terms of behavioral gains, as well as changes in sleep electrophysiology and brain activation (Cohen et al. 2005; Fischer et al. 2006; Maquet et al. 2000; Peigneux et al. 2000, 2003). However, more recent studies question the extent to which sleep plays a role in the consolidation of implicit MSL (Song et al. 2007; Nemeth et al. 2010; Hallgató et al. 2013; Meier and Cock 2014; Pan and Rickard 2015).

Importantly, neuroimaging evidence indicates that implicit MSL, similar to explicit MSL, also initially recruits hippocampal activity in addition to striatal network involvement (Willingham and Goedert-Eschmann 1999; Poldrack et al. 2001; Schendan et al. 2003; Cohen et al. 2005; Albouy et al. 2008). Whether implicit MSL can be behaviorally dissociated into egocentric versus allocentric sequence representations (as per explicit MSL) remains to be demonstrated, as does the nature of consolidation of such representations for implicit MSL (e.g., sleep versus time dependency and time-course). Thus, the focus of the current investigation was twofold: (1) to dissociate implicit MSL into its allocentric and egocentric representations, and (2) to explore the evolution of the consolidation of these distinct memory traces immediately after sleep or wake, and over the course of several days to disentangle what aspects of implicit MSL are sleep dependent, or not.

Specifically, we investigated whether a period of sleep compared with wake would differentially enhance performance of dissociable allocentric (spatial) and egocentric (motor)-referent cognitive representations of implicitly learned visual-motor sequences. We evaluated skill speed and the development of explicit sequence awareness as indices of consolidation. Another major aim was to ascertain whether the consolidation of the allocentric representation was associated with the sleep-dependent development of conscious awareness, as has previously been reported by others (see Fischer et al. 2006; Drosopoulos et al. 2011). Additional analyses also investigated the role of sleep on the generalization of these representations across hands.
We used a modified version of the SRTT, based on Albouy et al. (2013, 2015), whereby allocentric and egocentric representations could be behaviorally dissociated from an initial training sequence (Fig. 1). Awareness over time was assessed by a combination of selfreport, sequence recognition, and sequence generation tasks. To test sleep versus time-dependent effects of offline consolidation and awareness changes, measurements were taken before and after either a night of sleep, or across a day of wake, as well as $1 \mathrm{wk}$ after training. Electroencephalographic (EEG) recordings were taken during overnight intervals to characterize learning-dependent changes in sleep associated with changes in task performance (Fig. 2).

It was hypothesized that: (1) similar to explicit MSL, implicit sequence learning would result in behaviorally dissociable allocentric and egocentric components; (2) the allocentric representation would show sleep-dependent gains in performance speed, whereas the egocentric representation would show gains in performance speed across wake; (3) a night of sleep would reveal a development of explicit awareness of the implicitly learned sequence, and; (4) increases in allocentric representation performance and awareness post-sleep, would be associated with changes in post-learning sleep architecture. In particular, increases in sleep spindle characteristics such as spindle density, duration, and amplitude were hypothesized to be associated with improved performance, as previously reported for sleep-dependent consolidation of MSL (Morin et al. 2008; Albouy et al. 2013; Barakat et al. 2013) and other forms of procedural learning (Fogel and Smith 2006, 2011; Fogel et al. 2007; Tamminen et al. 2010).

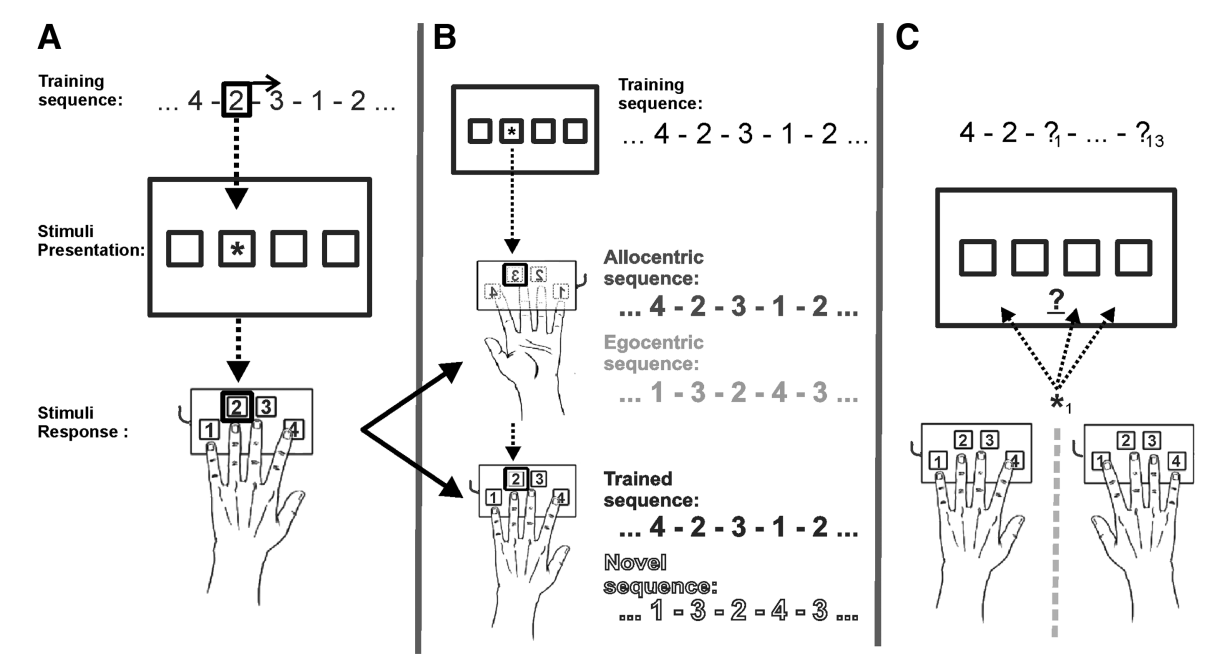

Figure 1. Behavioral tasks. $(A)$ Serial reaction time task (SRTT). A 12-item repeating pattern is determined by the locations of an onscreen asterisk cue. Participants must click the corresponding key as quickly and accurately as possible but are not told there is an underlying pattern guiding where the cue will appear. $(B)$ Representation testing. Relative to the training sequence, and with the trained hand, the SRTT was performed on each of four sequences. In two blocks the keypad was upside-down relative to training. In the Allocentric condition, the sequence of the onscreen cues were identical to training, preserving the spatial location of the finger presses of the learned sequence, but not the series of motor movements. Conversely, in the Egocentric block, the sequence of onscreen cues preserved the series of motor movements but not the spatial location of the finger presses. Two blocks were also performed with the keypad in the same orientation as training. One block was the same sequence as training and the other block was a different sequence. $(C)$ Generation task. After following two cues participants continued to create a sequence of 13 keypresses according to inclusion or exclusion instructions. Ability to create (inclusion) or withhold (exclusion) responses congruent with the training sequence indicated the accessibility and level of conscious awareness of learned sequence knowledge (Destrebecqz and Cleeremans 2001). The untrained (right) hand was also assessed with the generation task to evaluate whether allocentric or egocentric representations were preferentially involved in cross-hand generation skill. 


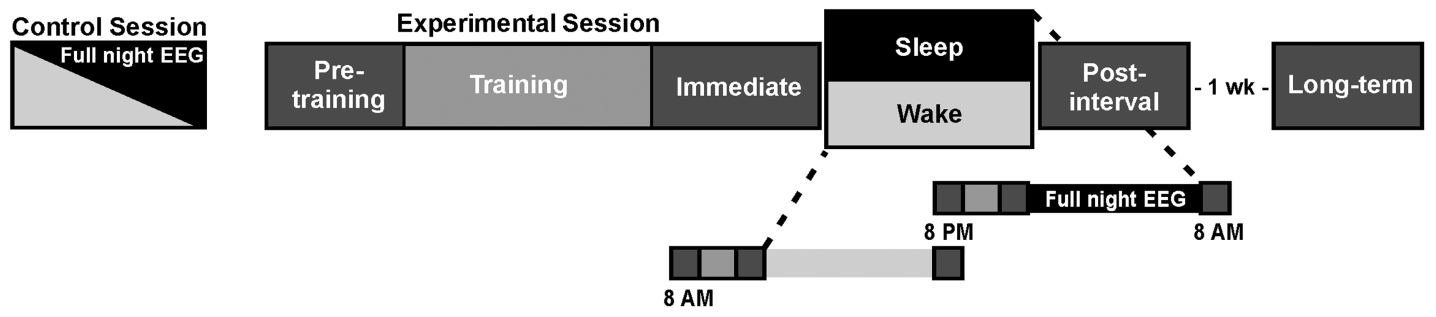

Figure 2. Experimental design. The Control session consisted of SRTT with no-learning (random cues) and a free generation task. Participants assigned to the Wake interval group completed the control session in the morning, whereas the Sleep group completed the control session in the late evening and had a baseline sleep EEG recording. The Experimental session consisted of a SRTT representation pretraining test, followed by SRTT training of a sequence over 13 blocks. Participants were then probed for sequence awareness. Following this, in the immediate post-training test, the generation tasks were followed by another SRTT representation test where participants then also provided sequence recognition ratings. Similarly, post-interval and long-term retest sessions consisted of the generation tasks followed by SRTT representation tests and recognition assessment. For the Wake condition, the experimental session and long-term test were performed in the morning, and the post-interval test in the evening. For the Sleep condition, the experimental session and long-term test were performed in the evening, and the post-interval test in the morning, with sleep EEG recorded between the experimental and the post-interval session. The long-term test session occurred exactly $1 \mathrm{wk}$ after the post interval session.

\section{RESULTS}

\section{SRTT training session}

As expected, error rates for control and experimental sessions were, in general, low ( $<5 \%$ of keypresses were incorrect), and groups did not differ in terms of chronotype, subjective or objective sleepiness or sleep quality (see Supplemental Materials, Control Measures). Also as expected, performance over the training session improved over blocks of SRTT practice, but not for the random control sequence, and did not differ between sleep/wake groups, as revealed by a Group (Sleep, Wake) $\times$ Session (Control, Training) $\times$ Block $(1-$ 13 blocks) ANOVA. This analysis yielded a significant Session $\times$ Block interaction, $F_{(12,408)}=2.22, P=0.039$, with no group effects (Blocks $\times$ Group, $F_{(12,408)}=0.40, P=0.964$; Session $\times$ Group, $F_{(1,34)}$ $=0.32, P=0.573$; Group, $\left.F_{(1,34)}=0.43, P=0.517\right)$. Thus, performance differed across blocks of practice as a function of practice on the Training session when compared with the Control session, irrespective of Sleep/Wake condition (Fig. 3A,B). Performance differed over the 13 training blocks for Control $\left(F_{(12,408)}=8.31, P<\right.$ $0.001)$ and Training sessions $\left(F_{(12,408)}=20.4, P<0.001\right)$. SRTT performance in the Training session consistently improved across blocks, whereas performance on the random control sequence, as expected, did not systematically change with practice. It is worth noting that a potential fatigue effect was apparent in the final block of the SRTT training session in both groups. However, this effect disappears, as is seen by the recovery of performance on the Trained sequence when tested after a brief rest interval at the immediate test session.

\section{SRTT representation tests}

\section{Pretraining versus immediate test}

To investigate how implicit learning of the Trained sequence impacted subsequent performance in the Sleep versus the Wake groups, a Representation (Egocentric, Allocentric, Trained, Novel) $\times$ Session (Pretraining, Immediate test $) \times$ Group (Sleep, Wake) mixed ANOVA was conducted on reaction times. We observed a significant interaction for Session and Representation $\left(F_{(3,102)}=33.76, \quad P<0.001, \quad \eta_{p}^{2}=0.498\right)$, but not for sleep/wake group $\left(F_{(1,34)}=0.01, \quad P=0.925\right)$ or any other interactions (Session $\times$ Group, $F_{(1,34)}=0.33, P=0.567$; Session $\times$ Representation, $F_{(3,102)}=1.68, P=0.177$; Group $\times$ Representation, $F_{(1,34)}=1.03, P=$ 0.384 ; Session $\times$ Representation $\times$ Group, $\left.F_{(3,102)}=0.47, P=0.707\right)$. Thus, as expected, performance from pretraining to the immediate test did not differ between Sleep/Wake groups. In addition, performance at the Immediate test differed between Representa- tion types, $F_{(3,35)}=66.11, P<0.001, \eta_{p}^{2}=0.654$. Egocentric $\left(t_{(35)}=\right.$ 5.81, $P<0.001=0.01)$, Allocentric $\left(t_{(35)}=8.87, P<0.001\right)$, and Trained $\left(t_{(35)}=11.40, P<0.001\right)$ conditions showed significant improvements in performance, while the Novel sequence did not significantly improve after training $\left(t_{(35)}=1.20, P=0.240\right.$; Fig. 3A,B). Importantly, the difference in performance between Trained and Novel sequence performance after training indicates that improvements were due to sequence-specific knowledge as opposed to general task effects. The difference in Egocentric and Allocentric performance after training indicates the successful acquisition and dissociation of these representations, as anticipated.

\section{Comparison of immediate, post-interval, and long-term tests}

To investigate changes in performance on the memory representation as a result of the intervening period of sleep or wake, a Group (Sleep, Wake) $\times$ Session (Immediate test, Post-interval, Long-term) $\times$ Representation (Egocentric, Allocentric, Trained, Novel) mixed ANOVA was used to analyze reaction times. A significant interaction was observed between Session and Representation $\left(F_{(6,204)}=2.89, P=0.014, \eta_{p}^{2}=0.078\right)$, but not for $\operatorname{Group}\left(F_{(1,34)}=\right.$ $0.05, P=0.842$ ) or other interactions (Representation $\times$ Group, $F_{(3,102)}=1.22, P=0.305$; Session $\times$ Group, $F_{(2,68)}=0.83, P=0.439$; Session $\times$ Representation $\times$ Group, $\left.F_{(6,204)}=0.34, P=0.915\right)$. Figure $3 \mathrm{~A}, \mathrm{~B}$ illustrates the similarity in performance between groups for each representation across sessions. Supplemental Table 2 summarizes the ANOVA results shown in Figure 3C,D.

Follow-up repeated-measures ANOVAs were performed individually on each representation block across the three sessions, Representation (Egocentric, Allocentric, Trained, Novel) $\times$ Session (Immediate, Post-interval, Long-term), to identify how performance on the different representations evolved over time. Egocentric performance was found to have a significant acrosssession improvement in reaction time performance $\left(F_{(2,70)}=\right.$ 9.66, $\left.P<0.001, \eta_{p}^{2}=0.216\right)$, but not the Allocentric $\left(F_{(2,70)}=\right.$ $0.07, P=0.915)$, Trained $\left(F_{(2,70)}=2.89, P=0.155\right)$, or Novel $\left(F_{(2,70)}=1.51, \quad P=0.229\right) \quad$ sequences. This improvement in Egocentric performance was apparent only after the week-long interval (from Post-interval test to Long-term test; $t_{(35)}=3.71, P=$ 0.001 ) but not across the shorter test interval (Immediate to Post-interval; $t_{(35)}=0.34, P=0.733$; Fig. 4). Surprisingly, this improvement in Motor performance was observed irrespective of Sleep or Wake condition (Fig. 4). Supplemental Table 2 summarizes performance across sessions for each of the SRTT representation blocks. 
A

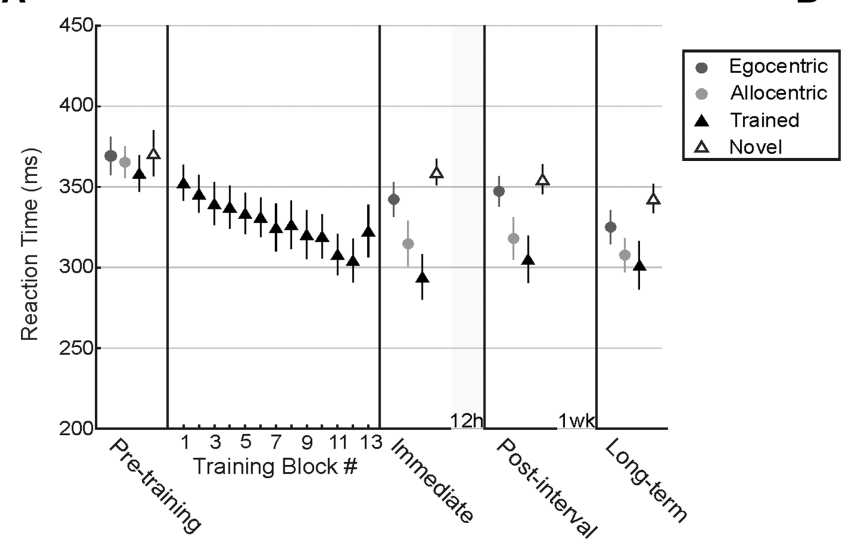

C

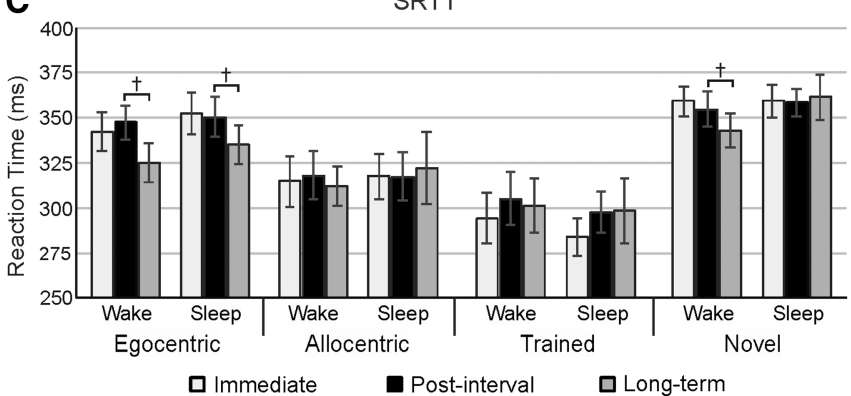

B

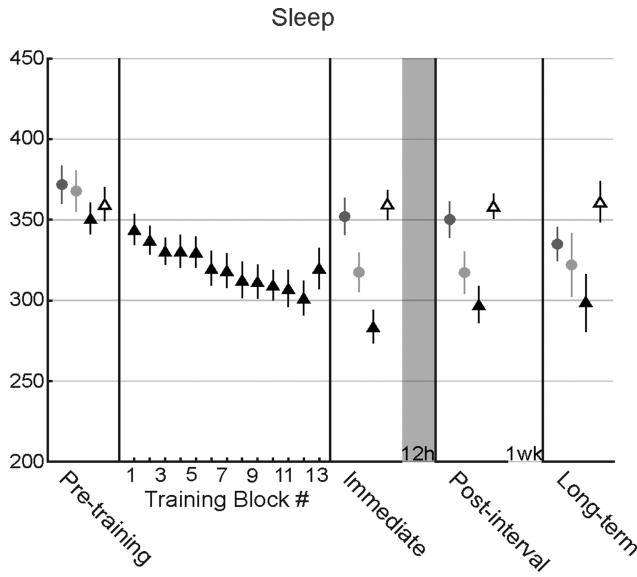

D

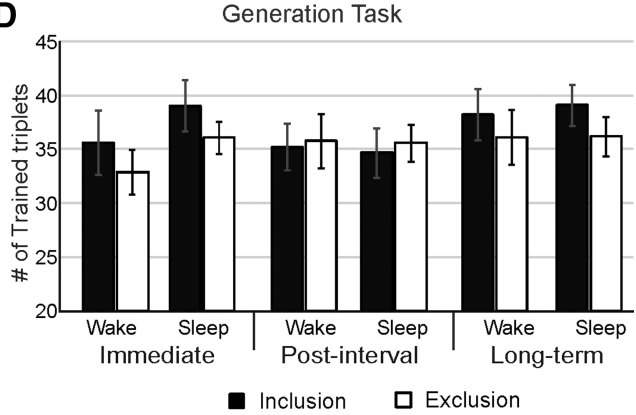

Figure 3. Overview of SRTT and generation task performance across sessions for intervals of wake and sleep. Four representations of sequence knowledge were tested with a SRTT block across four time points, with a training period of 13 blocks on one sequence. For the Wake group ( $A$ ) the interval between Immediate and Post-interval testing was a.m.-to-p.m., spent awake. For the Sleep group (B) the interval was p.m.-to-a.m., filled with sleep. (C) Compares the pattern across testing sessions of representation performance between Wake and Sleep groups. (D) Shows the generation task performance for Sleep and Wake groups. Reaction times are represented as the group averaged median response-times per block. Mixed ANOVA testing did not reveal any significant difference between Sleep and Wake group performance across SRTT or generation tasks. Error bars indicate standard error of the mean. For $(C)$ the ${ }^{\dagger}$ denotes significance of $P<0.05$ for within-group repeated-measures ANOVA.

\section{Similarity ratings}

To more comprehensively evaluate changes in memory, additional analyses aimed to investigate subjective assessments in recognition memory. Participants rated how similar each of the representation blocks felt compared with their training sequence during the representation testing. There was no significant difference between sleep/wake groups or across intervals. Only a main effect for representation, which upon following up revealed that the training sequence was identified as the most similar to training, followed by the allocentric, then egocentric sequence, with the novel sequence identified as the least similar (Supplemental Fig. 2). Details on the analyses, results, and discussion of this task can be found in the Supplemental Material.

\section{Trained hand sequence generation}

The level of implicit versus explicit memory performance was assessed at the Immediate, Post-interval, and Long-term tests by testing the subjects' ability to generate (Inclusion) or withhold generating (Exclusion) trained sequence responses. First, to verify that participants would respond using trained sequence knowledge, an ANOVA comparing training versus untrained (i.e., Novel) triplet generation was conducted. A significant effect for triplet type (Trained, Novel) was observed for both Inclusion $\left(F_{(1,34)}=36.10, P<0.001, \eta_{p}^{2}=0.515\right)$ and Exclusion instructions $\left(F_{(1,34)}=19.38, P<0.001, \eta_{p}^{2}=0.363\right)$, with no significant group or interaction effects. More Trained triplets were generated than Novel sequence triplets across all sessions. This indicates that the training sequence was selectively generated more than the untrained sequence, and verifies that the generation task engaged the trained memory trace.

Next, we conducted analyses directly comparing mean Trained triplet generation across instruction type. Reducing the number of Trained triplets generated under Exclusion instructions while increasing Trained triplet generation for Inclusion instructions indicates a shift from implicit to explicit consciousness awareness of a memory. By contrast, the inability to do so reflects that sequence knowledge is not under explicit control (i.e.,

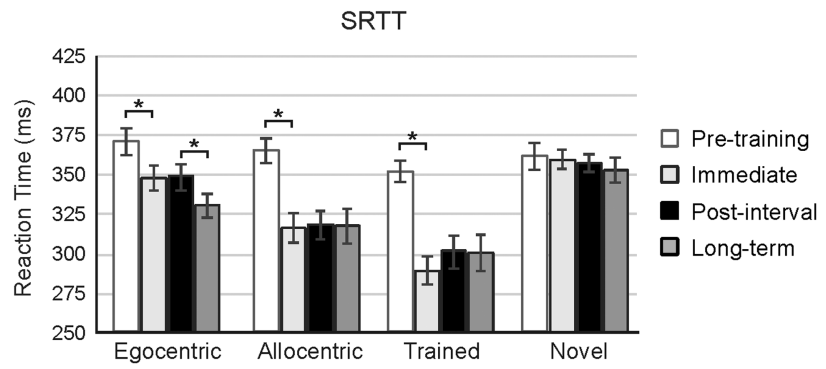

Figure 4. SRTT representation performance across the experiment Reaction times are block averaged median response-times. * denotes significance of $P<0.05$ for mixed within- and between-group ANOVA. No statistically reliable group difference was found between Wake and Sleep groups. Pooled group data are shown. 
sequence knowledge is implicit in nature; see Destrebecqz and Cleeremans 2001, and, Yonelinas and Jacoby 2012 for fully detailed accounts of this procedure). Our analyses did not indicate any significant difference in Trained triplet generation for instruction type (Inclusion versus Exclusion; $F_{(1,34)}=1.17, P=0.287$ ), between groups (Sleep versus Wake; $F_{(1,34)}=2.37, P=0.133$ ), across sessions (Immediate, Post-interval, Long-term; $F_{(2,68)}=1.93, P=$ $0.153)$, or interactions between these factors. In the event that Trained triplet generations occurred only within the first few keypresses after cueing, triplets were also analyzed for only the first, second, and third keypresses. This approach also did not reveal any significant group differences in responses or changes in performance across intervals. Together these results indicate that sequence knowledge remained implicit, for both groups, and across the entirety of the study (Supplemental Fig. 3A).

\section{Untrained-hand sequence generation}

Supplementary analyses were performed to investigate how effectively the Allocentric and Egocentric representations were transferred across hands, and whether this differed between groups and changed across the experimental intervals. Details on the analyses, results, and discussion of this task can be found in the Supplemental Material. Briefly, there was a significant effect for representation triplet type $\left(F_{(1,34)}=4.50, P=0.041\right)$ but not for Group $\left(F_{(1,34)}=0.73, P=0.400\right)$ or Session $\left(F_{(2,68)}=1.29, P=\right.$ $0.329)$. Significantly more Allocentric $\left(M_{ \pm S E}=35.87 \pm 0.12\right)$ than Egocentric triplets $(M \pm S E=33.28 \pm 1.17)$ were generated in the Inclusion instructions $(P=0.041)$. This suggests a small but stable response bias that favors accessing the allocentric representation for cross-hand sequence generation of implicitly learned motor sequences (Supplemental Fig. 3).

\section{Sleep EEG}

Paired $t$-tests were used to investigate changes in sleep architecture, across Control and Experimental nights. Surprisingly, this analysis revealed a significant increase in the proportion of REM sleep from the Control compared with the Experimental night (11.9\% REM increase; $\left.t_{(17)}=2.22, P=0.041\right)$. In contrast, the percentage of NREM stage 1 (NREM1; $\left.t_{(17)}=0.02, P=0.987\right)$, NREM2 $\left(t_{(17)}=\right.$ $1.62, P=0.123$ ), and NREM stage 3 (NREM3; $t_{(17)}=0.56, P=$ 0.583 ) sleep did not statistically differ between nights. Analysis of the spindle variables (peak amplitude, duration, peak frequency and density) did not reveal any significant differences between the Control and Experimental nights for slow (11-13.5 Hz), fast (13.5$16 \mathrm{~Hz}$ ) or total bandwidth (11-16 Hz) spindles (Supplemental Table 5). In addition, no sleep parameters were significantly correlated with the memory or awareness measures, after correction for multiple comparisons.

\section{Discussion}

While there is clear and substantive evidence demonstrating that sleep is important for declarative and explicit procedural motor sequence memory consolidation (Fischer et al. 2002; Robertson et al. 2004; Morin et al. 2008; Albouy et al. 2013, 2015; Debas et al. 2014 ), the role of sleep in implicit motor sequence memory consolidation has become increasingly unclear. The overall goal of the present study was to dissociate egocentric and allocentric representations from implicitly learned visual-motor sequences and investigate how these representations may benefit from sleep or wake in terms of consolidation over time. We successfully dissociated the allocentric and egocentric representations of an implicit motor sequence. The importance of this is twofold: First, it supports the notion that, similar to explicit MSL, allocentric and egocentric aspects of a learned sequence are acquired in dissociable representations via implicit learning - and perhaps in a similar manner as for explicit MSL. Second, this dissociation allowed us to evaluate the course of consolidation for these distinct representations over time, and to specifically test whether either aspect of an implicitly learned sequence is favorably enhanced by sleep or wake. Unlike explicit MSL, and contrary to our hypotheses, we did not find a benefit of sleep for the consolidation of the allocentric representation. Instead, surprisingly, irrespective of sleep or wake, we observed enhancement of the egocentric representation after a week-long interval. Overall, our results support the notion that sleep may not play a major role in enhancing memory consolidation of implicit motor learning; even when the skill memory is evaluated at the level of dissociable egocentric and allocentric component representations.

\section{Offline memory changes across retention intervals}

\section{Training sequence}

There are currently divergent findings as to whether a newly learned implicit motor sequence is enhanced over a period of sleep when compared with wake. Here, no significant differences on the SRTT training sequences were observed between sleepwake conditions, or across the post-training testing sessions (Immediate, Post-interval, Long-term). Reaction times were significantly and consistently fastest for the trained sequence compared with all other sequences tested. Thus, this pattern of results cannot be attributed to not having learned the sequence, or insufficient statistical power. Performance maintenance across the posttraining intervals suggests that the skill memory trace was stabilized, but not enhanced by an interval of sleep, wake, or even across a week's time (at Long-term retest). This result is consistent with previous studies investigating implicit MSL consolidation (Song et al. 2007; Nemeth et al. 2010; Meier and Cock 2014) also indicating equivalent performance stabilization as a function of either sleep or wake. These studies, however, reported offline gains in general skill performance across an interval of wake using a modified version of the SRTT (the Alternating SRTT) (Howard and Howard 1997). Using our approach, we did not observe significant increases in general skill. This was indicated by a lack of performance improvement on the untrained sequence across all testing. Overall, our results indicated that implicit MSL was stabilized independent of sleep or wake, and was specific to the learned sequence. Our results provide additional support for the extant literature (Robertson et al. 2004; Nemeth et al. 2010; Hallgató et al. 2013; Albouy et al. 2015) which suggests that explicit but not implicit motor sequence learning is enhanced by sleep when compared with wake.

\section{Allocentric and egocentric dissociation}

Immediately after training, both experimental groups successfully demonstrated that the trained sequence can be separated into both egocentric and allocentric representations, as acquired through implicit sequence learning. Similar to previous studies investigating explicit MSL consolidation (Albouy et al. 2013, 2015), the allocentric representation showed the most effective transfer as indicated by faster performance speed than for the egocentric sequence.

In addition, the extant literature suggests that the implicit versus explicit distinction may not be sufficient to explain whether sleep is involved in the consolidation process. In the present study, we sought to determine separating MSL into distinct allocentric and egocentric representations would help to resolve existing controversies about sleep-dependent implicit memory consolidation. Surprisingly, unlike explicit MSL, there was no sign of improvement across a night of sleep on the allocentric-referent 
performance. It is known that for explicit MSL, hippocampal activity is recruited during MSL learning, and that the allocentric representation, in particular, is related to hippocampal activation. This is thought to underlie the sleep-dependent gains of the allocentric sequence knowledge (Albouy et al. 2008, 2012, 2013, 2015). Like explicit MSL, there is also evidence that implicit learning also recruits the hippocampus (Schendan et al. 2003; Albouy et al. 2008). Despite these similarities and the successful behavioral dissociation of these representations, our results suggest that like explicit MSL, implicit MSL has dissociable allocentric and egocentric representations, but importantly, unlike explicit sequence learning, implicit allocentric memory consolidation is independent of sleep. Instead, our results of delayed enhancement of the egocentric representation (at Long-term testing) support the notion that for implicit MSL the sequence trace relies on the slower process of striatal consolidation, which does not strongly favor sleep or wake (Lehéricy et al. 2005).

Very few studies have looked beyond an interval of a couple days for SRTT consolidation (Romano et al. 2010; Drosopoulos et al. 2011; Meier and Cock 2014), and none have looked specifically at egocentric representation transfer. Because both periods of sleep and wake occupy the span of a week, unfortunately, it is not possible for us to identify the relative contributions of one state over the other, or if these gains were the product of striatal, motor cortical, or cerebellar networks known to be involved in implicit memory (Rauch et al. 1995, 1997; Doyon et al. 1996; Peigneux et al. 2000; Schendan et al. 2003; Lehéricy et al. 2005; Doyon et al. 2009). Neuroimaging studies using the same paradigm used here, would help to elucidate the relative roles of the hippocampus and striatum in implicit learning and consolidation that may not manifest themselves overtly at the behavioral level.

\section{Implicit versus explicit performance}

Contrary to our hypothesis, which were based on previous studies (Fischer et al. 2006; Drosopoulos et al. 2011) the results of our generation task did not indicate any sleep-dependent increases in explicit sequence awareness between groups or across sessions (see Supplemental Fig. 1), nor could subjects subjectively recognize the sequence (see Supplemental Fig. 2). Performance did not significantly differ between instructions to generate the sequence (inclusion condition), or to withhold generating the sequence (exclusion condition). Participants' ability to include elements of the trained sequence indicates sequence knowledge has been acquired. However, participants' inability to withhold generating components of the training sequence indicates an absence of conscious awareness of the trained sequence, which would have otherwise allowed them to control (i.e., withhold) generating the training sequence in accordance with task instruction. This paradigm is known as the process dissociation procedure (see Destrebecqz and Cleeremans 2001, and, Yonelinas and Jacoby 2012 for reviews of this method). Thus, skill knowledge appeared principally implicit rather than explicit, across the duration of the study.

That our study did not find sleep-dependent enhancement of explicit awareness comes contrary to Fischer et al. (2006) and Drosopoulos et al. (2011). This can be accounted for in a couple ways. Notably in Fischer et al.'s (2006) version of the generation task, the correct cue location was displayed following each generated response, whether the participants' responses were correct or not. This may have inadvertently developed a level of explicit sequence knowledge before the sleep interval; which consequently may have resulted in sleep further enhancing the explicit representation of the sequence as opposed to transforming implicit memory into explicit awareness. Unfortunately, it is unclear at this point how baseline levels of explicit awareness may or may not interact with sleep, and sleep-dependent consolidation of MSL (cf.
Robertson et al. 2004). It is possible, however, that even low or partial levels of explicit awareness may influence the cognitive and behavioral outcomes of implicit MSL consolidation. When Drosopoulos et al. (2011) sought to investigate this potential interaction between awareness and sleep, they found better generation task performance was independently associated with conditions of increased awareness and a period of sleep. They did not find an interaction between awareness manipulations and sleep. An important limitation of both studies (Fischer et al. 2006; Drosopoulos et al. 2011) is the absence of an exclusion condition in their generation tasks. Without using the process dissociation procedure (i.e., using both inclusion and exclusion tasks) the relative contributions of implicit versus explicit knowledge cannot be as precisely accounted for in generation task performance (see Destrebecqz and Cleeremans 2001; Destrebecqz et al. 2005; Yonelinas and Jacoby 2012; also see Materials and Methods for details).

These factors may be contributing to the discrepant findings seen across the implicit memory literature, especially concerning sleep's putative role in promoting insight into unconscious sequence knowledge. Here, we used multiple criteria for explicit knowledge, a restrictive awareness cut-off (see Supplemental Table 1), and the process dissociation procedure in a best effort to account for explicit awareness with high sensitivity. Importantly, with these measures and protocols in place, our results suggest that awareness for implicit motor sequence knowledge does not develop preferentially after a period of sleep or wake. Thus, our study provides support to the growing literature suggesting sleep does not play a role in the consolidation of implicitly learned MSL, especially in terms of promoting explicit insight.

\section{Sleep architecture and implicit memory consolidation}

To investigate whether implicit learning results in learningdependent changes in sleep, we compared baseline EEG sleep architecture from a control night (i.e., after a random sequence control task) to post-learning sleep. Surprisingly, we observed a significant increase in the duration of REM sleep, with no significant changes in any NREM sleep stages. Reports for REM effects related to MSL tend to vary (Peigneux et al. 2000, 2001; Fischer et al. 2002; Cohen et al. 2005; however, also, see Smith et al. 2004; Fischer et al. 2006; Pan and Rickard 2015). To our knowledge, only two other studies have found the amount of REM to be associated with sleep-dependent memory consolidation of SRTT implicit learning (Cajochen et al. 2004; Cohen et al. 2005), whereas others have found no evidence of sleep, REM or NREM, being involved in SRTT implicit learning (Fischer et al. 2006; Song et al. 2007; Nemeth et al. 2010). In the present study the increase in REM was not directly correlated with the performance of any of our memory measures. This may be due in-part to the observed stabilization rather than offline gains in performance. While speculative, our results are consistent with the notion that increased REM is thought to be a state of motor memory stabilization and integration (Maquet et al. 2000; Peigneux et al. 2003; Urbain et al. 2013; Debas et al. 2014), even without overt gains in SRTT training skill.

We also investigated whether spindle activity in NREM2 sleep plays a role in the consolidation of implicit MSL, as has been shown for explicit MSL (Fogel and Smith 2006, 2011; Morin et al. 2008; Albouy et al. 2013; Barakat et al. 2013). Contrary to our hypotheses, we did not observe learning-related changes in sleep spindles. These results are consistent with the notion that implicit MSL may undergo a different process of consolidation than explicit MSL.

\section{Conclusions}

This study has contributed to the ongoing debate surrounding the processes involved in learning and memory consolidation for 
implicit visual-motor skills. This was accomplished using a novel SRTT paradigm that allowed us to dissociate the allocentric and egocentric representations. Our results indicated that similar to explicit motor sequence learning, behaviorally dissociable allocentric and egocentric representations are acquired for implicitly learned visual-motor sequence learning. Similar to explicit memory, this likely reflects the involvement of distinct neural substrates. Contrary to our predictions, and unlike explicit memory, the allocentric representation was not specifically enhanced by sleep and did not recruit spindle activity. Notably, there was a consistent absence of sleep-dependent enhancement across the multiple types of tasks and measurements we used in this study. However, posttraining REM sleep duration increased when compared to the baseline sleep on the control night, which offers the possibility that sleep may still play a role in the consolidation and stabilization of the memory trace that was observed.

Across both experimental groups, we did observe enhancement of the egocentric representation when retested a week after initial training, which may reflect time-dependent (and sleep-independent) offline consolidation of egocentric sequence coordination. The exact mechanism underlying this change remains unresolved. In conclusion, our results provide evidence that when MSL is implicit, neither sleep nor wake preferentially contributes to the development of explicit awareness for implicitly learned visual-motor sequencing. The results of this study suggest that like explicit sequence learning, implicit sequence learning is comprised of distinct allocentric and egocentric representations. However, unlike explicit sequence learning, sleep does not preferentially enhance consolidation of the allocentric representation. Thus, our results suggest that implicit sequence consolidation takes place irrespective of sleep or wake, and importantly, that sleep is not recruited in all cases for the consolidation of allocentric memory representations.

\section{Materials and Methods}

\section{Participants}

All participants gave informed written consent. This research was approved by Western University's Research Ethics Board. Participants were compensated financially for study participation.

Participants between the ages of 20 and 35 were recruited through advertisements. An initial telephone interview was used to exclude participants for left-handedness (Edinburgh Handedness Inventory, Oldfield, 1971), hand mobility problems, atypical sleep patterns (sleep time outside the approximate hours of 10:00 p.m. to 9:00 a.m.), shift work, head injury, regular cigarette smoking, use of medications known to affect sleep, and history of chronic pain. In addition, participants with professional training as a musician or typist were excluded. Participants were required to abstain from drug use, caffeine, nicotine, and alcohol at least $3 \mathrm{~d}$ prior to, and throughout the duration of the study. Participants were also asked to keep consistent sleep routines throughout the study duration, which was confirmed by actigraphy and sleep diaries.

Participants who met the initial screening underwent a sleep disorder screening night, during which standard polysomnographic recordings (including electroencephalogram [EEG], electrooculogram [EOG], and electromyogram [EMG]; see Physiological Recordings) were obtained and subsequently analyzed for the presence of sleep disorders by a registered polysomnographic sleep technician. Additionally, in order to ensure normal sleep-wake patterns and rule out anxiety and depression, all participants were asked to fill out the Sleep Disorders Questionnaire (Douglass et al. 1994), as well as the Beck Depression (Beck et al. 1974) and Anxiety Inventories (Beck et al. 1988). For participants assigned to the experimental condition with multiple overnights in the laboratory, the screening night also served as an acclimatization night.
Fifty-one participants met the criteria for the study. Of this, eight participants dropped out before completion of the study. Three participants were excluded for failing to comply with the experimental protocol. Two participants did not complete the final retest session, but had completed the control and first experimental session. Their data were included for these sessions, with missing data from the final session substituted using multiple imputation. Analyses comparing the imputed data points versus list-wise exclusion of these participant did not yield any significant differences in the retest session results, indicating the pattern of results were unaffected by the imputation. Data from 40 participants (female $n=28)$ between 20 and $35 \mathrm{yr}$ of age $(M=22.9, \mathrm{SD}=3.3$ ) were included in the analyses. Nineteen were assigned to the overnight interval experimental condition (sleep interval), and 21 were assigned to the across-day (wake interval) testing condition. Of the total 40 participants, four (one assigned to the sleep interval and three assigned to wake interval) were identified as having developed explicit awareness of the sequence when assessed immediately after training (outlined in Supplemental Table 1). The data for these participants were removed from main experimental analyses, but were included in Supplemental Figure 1 to illustrate the difference in performance on the SRT when sequence knowledge was explicit versus implicit. Their ability to proficiently generate and withhold generating the training sequence immediately after training, compared with the remaining experimental sample, provides behavioral evidence that is consistent with explicit awareness of the sequence. Thus, the final analyses are reported from the remaining 36 participants who developed implicit but not explicit sequence knowledge at the end of training. Of this sample, 18 were in each of the Sleep interval condition and the Wake interval condition.

\section{Behavioral tasks}

\section{Serial reaction time task}

The Serial Reaction Time Task (SRTT) (Nissen and Bullemer 1987) is considered a classic implicit sequence learning paradigm. In the present study, learning performance was assessed using a modified version of the SRTT coded in MATLAB 2014a (Mathworks) using Psychtoolbox-3 (Brainard 1997; Kliener et al. 2007). The task (Fig. 1A) consisted of four horizontally arranged boxes on a black screen. Each key corresponded to the location of one of the squares onscreen, such that if the keypad was placed face up on top of the table and being handled with the left (nondominant) hand, the little finger would correspond to key 1 and to the leftmost box onscreen and the index finger would correspond to key 4 and the rightmost box onscreen. If the keypad was placed underneath the desk, oriented upside-down and being handled with the right hand, then the little finger would remain on key 1 but now correspond to the rightmost box onscreen, and the index finger would be on key 4 but correspond to the leftmost box onscreen. In this way, wherever the keypad was placed (upside or underneath the table) the leftmost key always corresponded to the leftmost box, and rightmost key to the rightmost box, maintaining spatial alignment between buttons and screen cues. With a 25 -sec rest interval before each block, directions were displayed onscreen instructing where to place the keypad and which hand to use (e.g., "Left Hand/ Keypad Up"). All participants were right-handed and completed the SRTT with their left hands. Participants were given a wrist support to prevent fatigue when completing blocks with the keypad on the table underside. Participants were video monitored to ensure compliance with the task instructions.

For the SRTT, participants were instructed to "respond as quickly and accurately as possible" to the appearance of an onscreen cue, by pressing the corresponding key to which square the cue appeared (Fig. 1A). The cue remained until one of the four keys were pressed, after which, the cue would disappear and then reappear in one of the four squares after a 120-msec interstimulus interval. Unbeknownst to the participants during SRTT training blocks, there was a repetitive underlying pattern determining the location of the cue. Auditory feedback was only given if the incorrect key was pressed, by the sounding of a short tone. 
The position where the cue appeared, the keypress, and the reaction time between cue onset and the keypress were recorded. Decreases in average reaction time across SRTT blocks of training on a repetitive sequence indicated the extent that sequence knowledge had been acquired. A Novel (i.e., untrained) sequence was administered to evaluate that reductions in reaction times were specific to the learned sequence as opposed to general task improvement.

\section{SRTT sequences}

Two second-order deterministic 12 -item sequences (Sequence $1=$ $3-4-2-3-1-2-1-4-3-2-4-1$; Sequence $2=3-4-1-2-4-3-1-4-2-1-$ $3-2$ ), and their mirror equivalents (respectively; Sequence $3=2$ 1-3-2-4-3-4-1-2-3-1-4; Sequence 4=2-1-4-3-1-2-4-1-3-4-23) were selected as the four possible sequences that could be assigned to train on. Each participant was assigned to only one of these sequences for 13 training blocks, with the keypad either on, or upside down, underneath the desk, for a total of eight sequence training conditions that any participant could be assigned to (four sequences, two keypad training locations). Every block of the SRTT was comprised of eight repetitions of the assigned 12-item sequences (96 cues per block), with the blocks starting at a random point within the given sequence. For these second-order sequences, the location of where the cue appeared was always determined by the previous two locations. The sequences did not include random items (as in some probabilistic modifications of SRTT; see Fu et al. 2008; Shanks et al. 2003), and thus, are considered deterministic. Sequences were balanced for frequency of cue location, frequency of transitions between locations, and did not contain back-to-back location repeats (e.g., 3-3) or consecutive "rolls" across all four keys (e.g., 4-3-2-1). Sequence 1 and Sequence 2 only differed in the second-order conditional structure such that each triplet within the sequence ended on a different location (e.g., Sequence $1=3-4-2 \ldots$, Sequence $2=3-4-1 \ldots)$. The sequences were selected for these qualities and used in previous studies (Reed and Johnson 1994; Destrebecqz and Cleeremans 2001; Fu et al. 2008).

\section{Control task}

The SRTT control session (i.e., no-learning) blocks were created by ordering eight randomly shuffled 12 -item sequences (96 cues per block), while controlling for back-to-back repeats, repetitions, distribution of keypresses, and across-key rolls. Congruent with the experimental SRTT training, the control task also consisted of 13 blocks. Participants also completed a generation task after control SRT training to establish chance levels of generating (to-be) trained sequence triplets (see Supplemental Table 1). There was a 7-d interval between the control session and the experimental session.

\section{SRTT representation testing}

The representation tests consisted of four different SRTT blocks (96 cues per block; 25-sec rest intervals between blocks), with each block repeating one of the following sequences: (1) A sequence assigned for repetitive training sequence ("Trained"), (2) an unfamiliar sequence ("Novel") which was the other equivalent sequence not assigned for training (i.e., if Trained $=$ Sequence 1 , Novel $=$ Sequence 2), (3) a spatially preserved, but not motor movement preserved sequence ("Allocentric") of the training sequence, and (4) a motoric but not spatially preserved sequence ("Egocentric") of the training sequence. Trained representation testing blocks utilized the same sequence as training with the keypad placed on the same side of the table as during SRTT training blocks. The Novel sequence blocks required the keypad to be in the same orientation as the assigned training condition, however the sequence throughout the block did not contain any of the same triplets as their assigned sequence. For Allocentric and Egocentric blocks, the keypad was placed up-side down, on the opposite side of the desk surface when compared with training (i.e., if training had the keypad table upside, then Allocentric/Egocentric would have the keypad placed table underside). For the Allocentric block, the cues appeared at the same spatial locations onscreen as the training sequence but the se- quence of finger movements was now different (i.e., inverted) because the key-to-location response contingencies were remapped to maintain spatial alignment (see Serial Reaction Time Task). For the Egocentric block, participants responded to a spatially inverted version of the sequence, which preserved the pattern of sequential finger movements as the training sequence but not the same onscreen spatial pattern (Fig. 1B).

The representation test blocks (Allocentric, Egocentric, Trained, Novel) isolate and probe the strength of different skill memory representations, with Allocentric and Egocentric preserving either the spatial or motor contingencies relative to the training sequence. In this manner, allocentric and egocentric representations could be dissociated by performance within the same hand and evaluated across retention intervals of time relative to the performance of trained and untrained sequences. The blocks of the representation testing were pseudo-randomly assigned so that the Trained block was tested third. This is in accord with typical SRTT transfer protocol (Destrebecqz and Cleeremans 2001) whereby after training, a transfer block of an alternative sequence is administered, which is then followed by administration of a block of the training sequence. This is done in order to demonstrate that increases in speed are specific to implicit learning of the trained sequence, and not due to general task practice effects.

\section{Awareness report}

Immediately following the training session of the SRTT participants were asked: "Do you believe that there was an underlying rule or pattern determining the training series." They were then asked, "How confident are you of this?" $(4=$ Not at all [0\%], $3=$ Unsure [25\%], 2 = Fairly Certain [75\%], $1=$ Absolutely Certain [100\%]). Pending the awareness probe, all participants were then informed that there was indeed a repeating sequence throughout the entirety of the training blocks. They were not, however, informed as to what the repeating sequence was. All participants who indicated "yes" to the belief of a pattern, with any degree of certainty, were asked to verbally describe and produce the training sequence (see Supplemental Table 1 for this breakdown). Those able to accurately recall $>36 \%$ of the training sequence, were excluded from analyzes $(n=4)$. These data are reported in the Supplemental Material (see Supplemental Fig. 1).

\section{Generation task}

Following the awareness report, participants were asked to generate a series of responses that were as similar as possible to the training sequences (i.e., "inclusion generation") as well as generate a series of responses that were as explicitly different as possible to the training series (i.e., "exclusion generation"). The generation blocks consisted of two initial cues that the participant had to respond to as in the typical SRTT, by pressing the corresponding key where the cue appeared, then proceed to create a series of 13 subsequent responses congruent with the instructions of that block (inclusion versus exclusion; Fig. 1C). Each block consisted of seven sets of these series generations, with a different cue pair starting off each set.

In both inclusion and exclusion generation blocks, participants were explicitly told to avoid generating in the same location twice (or more) in a row, not to make rolling transitions across the keypad (i.e., 4-3-2-1, 1-2-3-4), to be as nonrepetitive as possible within response sets (e.g., avoid making a response like 3-2-1-3$2-1$ ), and encouraged to make choices that came most naturally to them. Participants were also informed that this section of the experiment was not timed. Inclusion and exclusion blocks were performed by both right and left hands, with the keypad in the same orientation as training, for one inclusion and one exclusion block per hand. The generation task was performed at the immediate, post-interval, and long-term testing phases.

The inclusion and exclusion blocks performed by the trained (i.e., left) hand together are considered a process dissociation procedure (Jacoby 1991; Destrebecqz and Cleeremans 2001; Yonelinas and Jacoby 2012); a technique used to assess the extent that learned sequence knowledge exists as conscious versus unconscious knowledge. Under inclusion instructions, the amount of 
sequence responses congruent with the assigned training sequence is taken to indicate the level of explicit awareness of the sequence. However, as Destrebecqz and Cleeremans (2001) have previously noted, the inclusion generation score does not purely measure explicit awareness of the sequence and is contaminated by the influence of implicit knowledge. On the other hand, the exclusion block is thought to be a direct measure of implicit knowledge. If the participant generates sequence triplets congruent with the training sequence, despite given directions not to, this indicates that they have acquired the knowledge but cannot exert conscious control over it. Thus, taken together, the inclusion and exclusion tasks provide a more sensitive measure of how explicit the sequence knowledge is at a given time point throughout the study, than purely inclusion generation instructions.

The generation task for the control session consisted of only two blocks, one per hand, with the keypad in the same orientation as the preceding baseline session SRTT. The control session generation task only differed in that the participants were naive to the testing and training sequences and so instead were asked to generate their own novel sequence. The amount of trained sequence triplets generated here, while the participants were still naïve to their training sequences, was used to calculate experiment-wise chance for screening (see Supplemental Table 1).

\section{Similarity report}

Participants gave similarity ratings after post-training SRTT representation tests as an index of sequence representation recognition on a four-point Likert rating ( $4=$ Nothing in common, $3=$ Mostly Dissimilar, 2 = Very Similar, 1 = Identical). Further details can be found in the Supplemental Materials section (see Supplemental Fig. 2, Similarity ratings and recognition memory).

\section{Psychomotor vigilance task}

The PVT was used as an objective measure of sustained vigilance (Dinges and Powell 1985). The PVT is a simple reaction time test, whereby participants must respond as quickly as possible to a visual cue presented at a random interval (between 2 and $10 \mathrm{sec}$ ) with a key press. Participants performed 60 trials, taking approximately 8 min to complete. The PVT is used in this study to indicate vigilance at different times during the day and across experimental sessions.

\section{Physiological recordings}

Embla Titanium (Natus) polysomnographic (PSG) systems were used to perform in-laboratory sleep recordings. Physiological data were recorded at a sampling rate of $512 \mathrm{~Hz}$, with a high pass filter $=0.1 \mathrm{~Hz}$ and low pass filter $=220 \mathrm{~Hz}$. EEG, electrooculogram (EOG), and electromyogram (EMG) recordings were taken using gold-plated electrodes applied to the skin. EEG and EOG (from the left and right outer canthus of the eye) were recorded and re-referenced offline to mastoid derivations (M1 and M2). The EMG (submental chin muscles) channel was recorded as a bipolar derivation. Scalp electrodes were placed according to the international 10-20 system. Sleep stages were manually scored in 30-sec intervals by an expert registered polysomnographic technologist, in accordance with standard sleep scoring criteria (Iber et al. 2007) using RemLogic software (Natus).

\section{Screening night}

The screening night recordings included EMG, EOG, and EEG electrodes on the face and scalp (locations: Fz, Cz, Pz, Oz, M1, M2), as well as PSG measurements of respiration (via thorax and abdomen respiratory belts), electrocardiographic activity (via electrodes placed on the surface of the skin below each clavicle), leg muscle activity (via electrodes placed on the surface of the skin on the anterior tibialis muscle of each leg), and blood oxygen saturation (via a finger probe placed on the index finger of the left hand). Recordings were scored by an expert registered polysomnographic technician according to clinical scoring guidelines (Iber et al. 2007).

\section{Overnight experimental EEG}

The montage included EMG, EOG, and EEG electrodes placed at F3, Fz, F4, C3, Cz, C4, P3, Pz, P4, Oz, M1, and M2. Detection of sleep spindles was performed using in-house EEGlab (Delorme and Makeig, 2004) compatible software (https://github.com/ stuartfogel/detect spindles) written for Matlab R2014a (Mathworks Inc.). The data for one participant's control night was corrupt, thus, these analyses were run on $N=17$ participants. The spindle detection was performed at $\mathrm{Fz}$ for slow spindles (11-13.5 $\mathrm{Hz})$, Pz for fast spindles (13.5-16 Hz), and $\mathrm{Cz}$ for total bandwidth spindles $(11-16 \mathrm{~Hz})$. Fast and slow spindles were categorized so that they were orthogonal at the scalp locations where they predominate topographically (Jobert et al. 1992; Werth et al. 1997; Zeitlhofer et al. 1997). EEG data were initially down-sampled to $128 \mathrm{~Hz}$ and extracted from movement artifact-free, NREM2 sleep epochs. The detection method (Fogel et al. 2014; Ray et al. 2015) used a complex demodulation transformation of the EEG signal with a bandwidth of $5 \mathrm{~Hz}$ centered about a carrier frequency of $13.5 \mathrm{~Hz}$ (i.e., 11-16 Hz). Each data point was transformed to $Z$-scores using the mean and the standard deviation derived from a 60-sec sliding window. Events (spindle onsets, peaks and offsets) were then detected on the transformed signal with a $z$-score threshold of $z=2.33$. The variables of interest extracted from this method include spindle peak amplitude, spindle duration, peak frequency and spindle density (number of spindles per-minute of NREM sleep).

\section{Acknowledgments}

We thank Julien Doyon and Genevieve Albouy for the foreground and inspiration that led to the research ideas investigated here. This research was funded by a Canada Excellence Research Chair (CERC) grant to author A.M.O., with scholarship support to author V.S. from an Ontario Graduate Scholarship, and a grant to author S. M.F. from the Natural Sciences and Engineering Research Council of Canada.

\section{References}

Albouy G, Sterpenich V, Balteau E, Vandewalle G, Desseilles M, Dang-Vu TT, Darsaud A, Ruby P, Luppi P-H, Degueldre C, et al. 2008. Both the hippocampus and striatum are involved in consolidation of motor sequence memory. Neuron 58: 261-272.

Albouy G, Sterpenich V, Vandewalle G, Darsaud A, Gais S, Rauchs G, Desseilles M, Boly M, Dang-Vu TT, Balteau E, et al. 2012. Neural correlates of performance variability during motor sequence acquisition. Neuroimage 60: 324-331.

Albouy G, Fogel SM, Pottiez H, Nguyen VA, Ray L, Lungu O, Carrier J, Robertson EM, Doyon J. 2013. Daytime sleep enhances consolidation of the spatial but not motoric representation of motor sequence memory. PLoS One 8: e52805.

Albouy G, Fogel SM, King BR, Laventure S, Benali H, Karni A, Carrier J, Robertson EM, Doyon J. 2015. Maintaining vs. enhancing motor sequence memories: respective roles of striatal and hippocampal systems. Neuroimage 108: 423-434.

Barakat M, Carrier J, Debas K, Lungu O, Fogel SM, Vandewalle G, Hoge RD, Bellec P, Karni A, Ungerleider LG, et al. 2013. Sleep spindles predict neural and behavioral changes in motor sequence consolidation. Hum Brain Mapp 34: 2918-2928.

Beck AT, Epstein N, Brown G, Steer RA. 1988. An inventory for measuring clinical anxiety: psychometric properties. J Consult Clin Psychol 56: 893897

Beck AT, Rial WY, Rickels K. 1974. Short form of depression inventory: crossvalidation. Psychol Rep 34: 1184-1186.

Bergmann TO, Mölle M, Marshall L, Kaya-Yildiz L, Born J, Roman Siebner H. 2008. A local signature of LTP- and LTD-like plasticity in human NREM sleep. Eur J Neurosci 27: 2241-2249.

Brainard DH. 1997. The psychophysics toolbox. Spat Vis 10: 433-436.

Cajochen C, Knoblauch V, Wirz-Justice A, Kräuchi K, Graw P, Wallach D. 2004. Circadian modulation of sequence learning under high and low sleep pressure conditions. Behav Brain Res 151: 167-176.

Cohen DA, Pascual-Leone A, Press DZ, Robertson EM. 2005. Off-line learning of motor skill memory: a double dissociation of goal and movement. Proc Natl Acad Sci 102: 18237-18241.

Debas K, Carrier J, Barakat M, Marrelec G, Bellec P, Tahar AH, Karni A, Ungerleider LG, Benali H, Doyon J. 2014. Off-line consolidation of 
motor sequence learning results in greater integration within a cortico-striatal functional network. Neuroimage 99: 50-58.

Delorme A, Makeig S. 2004. EEGLAB: An open source toolbox for analysis of single-trial EEG dynamics including independent component analysis. $J$ Neurosci Methods 134: 9-21.

Destrebecqz A, Cleeremans A. 2001. Can sequence learning be implicit? New evidence with the process dissociation procedure. Psychon Bull Rev 8: $343-350$.

Destrebecqz A, Peigneux P, Laureys S, Degueldre C, Del Fiore G, Aerts J, Luxen A, Van Der Linden M, Cleeremans A, Maquet P. 2005. The neural correlates of implicit and explicit sequence learning: interacting networks revealed by the process dissociation procedure. Learn Mem 12: 480-490.

Dinges DF, Powell JW. 1985. Microcomputer analyses of performance on a portable, simple visual RT task during sustained operations. Behav Res Methods Instrum Comput 17: 652-655.

Douglass AB, Bornstein RF, Nino-Murcia G, Keenan S, Miles L, Zarcone VP Jr, Guilleminault C, Dement WC. 1994. The sleep disorders questionnaire: I. creation and multivariate structure of SDQ. Sleep 17: 160.

Doyon J, Owen AM, Petrides M, Sziklas V, Evans AC. 1996. Functional anatomy of visuomotor skill learning in human subjects examined with positron emission tomography. Eur J Neurosci 8: 637-648.

Doyon J, Bellec P, Amsel R, Penhune V, Monchi O, Carrier J, Lehéricy S, Benali H. 2009a. Contributions of the basal ganglia and functionally related brain structures to motor learning. Behav Brain Res 199: 61-75.

Doyon J, Korman M, Morin A, Dostie V, Hadj Tahar A, Benali H, Karni A, Ungerleider LG, Carrier J. 2009b. Contribution of night and day sleep vs. simple passage of time to the consolidation of motor sequence and visuomotor adaptation learning. Exp Brain Res 195: 15-26.

Drosopoulos S, Wagner U, Born J. 2005. Sleep enhances explicit recollection in recognition memory. Learn Mem 12: 44-51.

Drosopoulos S, Harrer D, Born J. 2011. Sleep and awareness about presence of regularity speed the transition from implicit to explicit knowledge. Biol Psychol 86: 168-173.

Fischer S, Hallschmid M, Elsner AL, Born J. 2002. Sleep forms memory for finger skills. Proc Natl Acad Sci 99: 11987-11991.

Fischer S, Drosopoulos S, Tsen J, Born J. 2006. Implicit learning-explicit knowing: a role for sleep in memory system interaction. J Cogn Neurosci 18: $311-319$.

Fogel SM, Smith CT. 2006. Learning-dependent changes in sleep spindles and Stage 2 sleep. J Sleep Res 15: 250-255.

Fogel SM, Smith CT. 2011. The function of the sleep spindle: a physiological index of intelligence and a mechanism for sleep-dependent memory consolidation. Neurosci Biobehav Rev 35: 1154-1165.

Fogel SM, Smith CT, Cote KA. 2007. Dissociable learning-dependent changes in REM and non-REM sleep in declarative and procedural memory systems. Behav Brain Res 180: 48-61.

Fogel SM, Albouy G, Vien C, Popovicci R, King BR, Hoge R, Jbabdi S, Benali H, Karni A, Maquet P. 2014. fMRI and sleep correlates of the age-related impairment in motor memory consolidation. Hum Brain Mapp 35: 3625-3645.

Fogel SM, Ray L, Binnie L, Owen AM. 2015. How to become an expert: a new perspective on the role of sleep in the mastery of procedural skills. Neurobiol Learn Mem 125: 236-248.

$\mathrm{Fu} \mathrm{Q}, \mathrm{Fu}$ X, Dienes Z. 2008. Implicit sequence learning and conscious awareness. Conscious Cogn 17: 185-202.

Giuditta A. 2014. Sleep memory processing: the sequential hypothesis. Front Syst Neurosci 8: 219.

Gómez RL, Bootzin RR, Nadel L. 2006. Naps promote abstraction in language learning infants. Psychol Sci 17: 670-674.

Hallgató E, Györi-Dani D, Pekár J, Janacsek K, Nemeth D. 2013. The differential consolidation of perceptual and motor learning in skill acquisition. Cortex 49: 1073-1081.

Howard JH Jr, Howard DV. 1997. Age differences in implicit learning of higher order dependencies in serial patterns. Psychol Aging 12: 634-656.

Iber C, Ancoli-Israel S, Chesson AL Jr, Quan SF. 2007. The AASM Manual for the Scoring of Sleep and Associated Events: Rules Terminology and Technical Specifications 1st ed.

Jacoby LL. 1991. A process dissociation framework: separating automatic from intentional uses of memory. J Mem Lang 30: 513-541.

Jobert M, Poiseau E, Jahnig P, Schulz H, Kubicki S. 1992. Topographical analysis of sleep spindle activity. Neuropsychobiology 26: 210-217.

Keisler A, Ashe J, Willingham DT. 2007. Time of day accounts for overnight improvement in sequence learning. Learn Mem 14: 669-672.

Kleiner M, Brainard D, Pelli D. 2007. What's new in psychtoolbox-3? Perception 36: ECVP Abstract Supplement.

Korman M, Raz N, Flash T, Karni A. 2003. Multiple shifts in the representation of a motor sequence during the acquisition of skilled performance. Proc Natl Acad Sci 100: 12492-12497.

Lehéricy S, Benali H, Van De Moortele P-F, Peiegrini-Issac M, Waechter T, Ugurbil K, Doyon J, Ungerleider LG. 2005. Distinct basal ganglia territories are engaged in early and advanced motor sequence learning. Proc Natl Acad Sci 102: 12566-12571.

Maquet P. 2001. The role of sleep in learning and memory. Science 294: 1048-1052.

Maquet P, Laureys S, Peigneux P, Fuchs S, Petiau C, Phillips C, Aerts J, Del Fiore G, Degueldre C, Meulemans T, et al. 2000. Experience-dependent changes in cerebral activation during human REM sleep. Nat Neurosci 3: 831-6.

Meier B, Cock J. 2014. Offline consolidation in implicit sequence learning. Cortex 57: 156-166.

Morin A, Doyon J, Dostie V, Barakat M, Hadj Tahar A, Korman M, Benali H, Karni A, Ungerleider LG, Carrier J. 2008. Motor sequence learning increases sleep spindles and fast frequencies in post-training sleep. Sleep 31: $1149-56$.

Nemeth D, Janacsek K, Londe Z, Ullman MT, Howard DV, Howard JH. 2010. Sleep has no critical role in implicit motor sequence learning in young and old adults. Exp Brain Res 201: 351-358.

Nemeth D, Csabi E, Janacsek K, Varszegi M, Mari Z. 2012. Intact implicit probabilistic sequence learning in obstructive sleep apnea. J Sleep Res 21: 396-401.

Nissen MJ, Bullemer P. 1987. Attentional requirements of learning: evidence from performance measures. Cogn Psychol 19: 1-32.

Oldfield RC. 1971. The assessment and analysis of handedness: the Edinburgh inventory. Neuropsychologia 9: 97-113.

Pan SC, Rickard TC. 2015. Sleep and motor learning: is there room for consolidation? Psychol Bull 141: 812-834.

Payne JD, Schacter DL, Propper RE, Huang LW, Wamsley EJ, Tucker MA, Walker MP, Stickgold R. 2009. The role of sleep in false memory formation. Neurobiol Learn Mem 92: 327-334.

Peigneux P, Maquet P, Meulemans T, Destrebecqz A, Laureys S, Degueldre C, Del Fiore G, Aerts J, Luxen A, Franck G, et al. 2000. Striatum forever, despite sequence learning variability: a random effect analysis of PET data. Hum Brain Mapp 10: 179-194.

Peigneux P, Laureys S, Delbeuck X, Maquet P. 2001. Sleeping brain, learning brain. The role of sleep for memory systems. Neuroreport 12: A111-A124.

Peigneux P, Laureys S, Fuchs S, Destrebecqz A, Collette F, Delbeuck X, Phillips C, Aerts J, Del Fiore G, Degueldre C, et al. 2003. Learned material content and acquisition level modulate cerebral reactivation during posttraining rapid-eye-movements sleep. Neuroimage 20: $125-134$.

Peigneux P, Laureys S, Fuchs S, Collette F, Perrin F, Reggers J, Phillips C, Degueldre C, Del Fiore G, Aerts J, et al. 2004. Are spatial memories strengthened in the human hippocampus during slow wave sleep? Neuron 44: 535-545.

Poldrack RA, Clark J, Pare-Blagoev EJ, Shohamy D, Moyano JC, Meyers C, Gluck MA. 2001. Interactive memory systems in the human brain Nature 414: 546-550.

Rasch B, Born J. 2013. About sleep's role in memory. Physiol Rev 93: 681-766.

Rauch SL, Savage CR, Brown HD, Curran T, Alpert NM, Kendrick A, Fischman AJ, Kosslyn SM. 1995. A PET investigation of implicit and explicit sequence learning. Hum Brain Mapp 3: 271-286.

Rauch SL, Whalen PJ, Savage CR, Curran T, Kendrick A, Brown HD, Bush G, Breiter HC, Rosen BR. 1997. Striatal recruitment during an implicit sequence learning task as measured by functional magnetic resonance imaging. Hum Brain Mapp 5: 124-132.

Ray L, Sockeel S, Soon M, Bore A, Myhr A, Stojanoski B, Cusack R, Owen AM, Doyon J, Fogel SM. 2015. Expert and crowd-sourced validation of an individualized sleep spindle detection method employing complex demodulation and individualized normalization. Front Hum Neurosci 9: $1-16$.

Reed J, Johnson P. 1994. Assessing implicit learning with indirect tests: determining what is learned about sequence structure. Journal of Exp Psychol Learn Mem Cogn 20: 585-594.

Robertson EM, Pascual-Leone A, Press DZ. 2004. Awareness modifies the skill-learning benefits of sleep. Curr Biol 14: 208-212.

Romano JC, Howard JH, Howard DV. 2010. One-year retention of general and sequence-specific skills in a probabilistic, serial reaction time task. Memory 18: $427-441$

Schendan HE, Searl MM, Melrose RJ, Stern CE. 2003. An fMRI study of the role of the medial temporal lobe in implicit and explicit sequence learning. Neuron 37: 1013-1025.

Shanks DR, Wilkinson S, Channon S. 2003. Relationship between priming and recognition in deterministic and probabilistic sequence learning. $J$ Exp Psychol Learn, Mem, Cognit 29: 248-261.

Sirota A, Buzsáki G. 2007. Interaction between neocortical and hippocampal networks via slow oscillations. Thalamus Relat Syst 3: 245 .

Smith CT. 2001. Sleep states and memory processes in humans: procedural versus declarative memory systems. Sleep Med Rev 5: 491-506. 
Smith CT, Aubrey JB, Peters KR. 2004. Different roles for REM and stage 2 sleep in motor learning: a proposed model. Psychol Bull 44: 79-102.

Song S, Howard JH, Howard DV. 2007. Sleep does not benefit probabilistic motor sequence learning. Exp Brain Res 27: 12475-12483.

Squire LR. 2009. Memory and Brain Systems: 1969-2009. J Neurosci 29: 12711-12716.

Stickgold R. 2013. Parsing the role of sleep in memory processing. Curr Opin Neurobiol 23: 847-853.

Stickgold R, Walker MP. 2013. Sleep-dependent memory triage: evolving generalization through selective processing. Nat Neurosci 16: 139-145.

Takehara-Nishiuchi K, McNaughton BL. 2008. Spontaneous changes of neocortical code for associative memory during consolidation. Science 322: 960-963.

Tamminen J, Payne JD, Stickgold R, Wamsley EJ, Gaskell MG. 2010. Sleep spindle activity is associated with the integration of new memories and existing knowledge. I Neurosci 30: 14356-14360.

Tononi G, Cirelli C. 2014. Sleep and the price of plasticity: from synaptic and cellular homeostasis to memory consolidation and integration. Neuron 81: 12-34.

Urbain C, Schmitz R, Schmidt C, Cleeremans A, Van Bogaert P, Maquet P, Peigneux P. 2013. Sleep-dependent neurophysiological processes in implicit sequence learning. J Cogn Neurosci 25: 2003-2014.
Wagner U, Gais S, Haider H, Verleger R, Born J. 2004. Sleep inspires insight. Nature 427: 352-355.

Walker MP. 2005. A refined model of sleep and the time course of memory formation. Behav Brain Sci 28: 51-64.

Werth E, Achermann P, Dijk D, Borbély A. 1997. Spindle frequency activity in the sleep EEG: individual differences and topographic distribution. Clin Neurophysiol 103: 535-542.

Willingham DB, Goedert-Eschmann K. 1999. The relation between implicit and explicit learning: evidence for parallel development. Psychol Sci 10: 531-534.

Yonelinas AP, Jacoby LL. 2012. The process-dissociation approach two decades later: convergence, boundary conditions, and new directions. Mem Cognit 40: 663-680.

Yordanova J, Kolev V, Verleger R, Bataghva Z, Born J, Wagner U. 2008. Shifting from implicit to explicit knowledge: different roles of early- and late-night sleep. Learn Mem 15: 508-515.

Zeitlhofer J, Gruber G, Anderer P, Asenbaum S, Schimicek P, Saletu B. 1997. Topographic distribution of sleep spindles in young healthy subjects. $J$ Sleep Res 6: 149-155.

Received November 29, 2016; accepted in revised form October 3, 2017. 


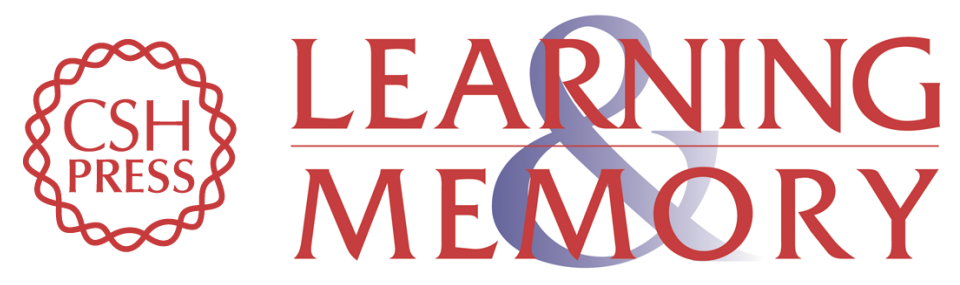

Does sleep facilitate the consolidation of allocentric or egocentric representations of implicitly learned visual-motor sequence learning?

Jeremy Viczko, Valya Sergeeva, Laura B. Ray, et al.

Learn. Mem. 2018, 25:

Access the most recent version at doi:10.1101/lm.044719.116

Supplemental http://learnmem.cshlp.org/content/suppl/2018/01/12/25.2.67.DC1
Material

References This article cites 81 articles, 12 of which can be accessed free at: http://learnmem.cshlp.org/content/25/2/67.full.html\#ref-list-1

Creative This article is distributed exclusively by Cold Spring Harbor Laboratory Press for the Commons first 12 months after the full-issue publication date (see

License http://learnmem.cshlp.org/site/misc/terms.xhtml). After 12 months, it is available under a Creative Commons License (Attribution-NonCommercial 4.0 International), as described at http://creativecommons.org/licenses/by-nc/4.0/.

Email Alerting Receive free email alerts when new articles cite this article - sign up in the box at the Service top right corner of the article or click here. 